\title{
DEVELOPMENT OF NON-CELLULOSIC INSULATION
}

FOR TRANSFORMERS

\author{
DOE Contract Ilo. DE-AC02-79ET29343 \\ QUARTERLY REPORT INO, 2 \\ APRIL, 1980
}

\author{
PREPARED BY: \\ W.A. FESSLER \\ Principal InVestigator \\ General Electric Company \\ Large Transformer Business Division \\ TECHNICAL RESOURCES OPERATION \\ PitTsfield, llassachusetts
}

\author{
PREPARED FOR: \\ J. VORA \\ Project Mlanager \\ U.S. Department of Energy \\ WASHINGTON, D.C.
}

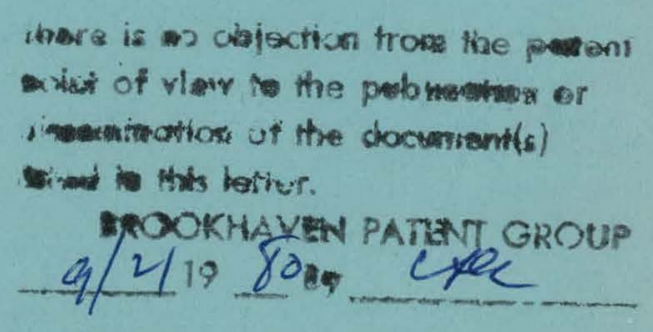




\section{DISCLAIMER}

This report was prepared as an account of work sponsored by an agency of the United States Government. Neither the United States Government nor any agency Thereof, nor any of their employees, makes any warranty, express or implied, or assumes any legal liability or responsibility for the accuracy, completeness, or usefulness of any information, apparatus, product, or process disclosed, or represents that its use would not infringe privately owned rights. Reference herein to any specific commercial product, process, or service by trade name, trademark, manufacturer, or otherwise does not necessarily constitute or imply its endorsement, recommendation, or favoring by the United States Government or any agency thereof. The views and opinions of authors expressed herein do not necessarily state or reflect those of the United States Government or any agency thereof. 


\section{DISCLAIMER}

Portions of this document may be illegible in electronic image products. Images are produced from the best available original document. 
Master

General Electric Ref. No. CCR-80-07

DEVELOPMENT OF NON-CELLULOSIC INSULATION

FOR TRANSFORMERS

DOE CONTRACT 110, DE-ACO2-79ET29343

QUARTERLY REPORT WO, 2

APRIL, 1980

PREPARED BY:

W.A. FESSLER

PRINCIPAL INVESTIGATOR

General Electric Company

LARGE TRANSFORMER BUSINESS DIVISION TECHNICAL RESOURCES OPERATION

PitTsfield, llassachusetTs

PREPARED FOR:

J. NORA

Project Manager

U.S. DEPARTMENT OF EnERgY

WASHINGTON, DEC.

GENERAL ELECTRIC

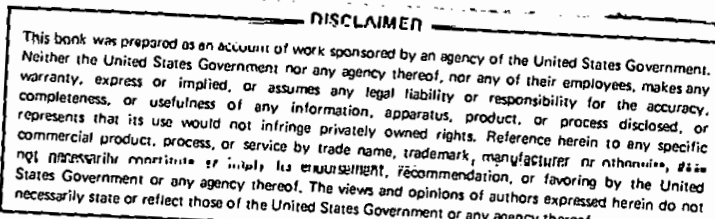

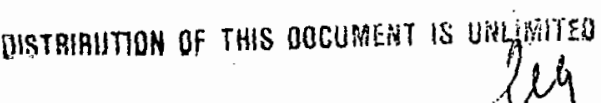




\section{CONTENTS}

$\underline{\text { Page }}$

SUMMARY OF PROGRAM .....................

TASK 1.0 DEVELOPMENT OF PARAMETRIC MODEL FOR SOLID INSULATION IN TRANSFORMERS. ............. $1-1$ Progress ................ . . . . . . . Future Activity. . . . . . . . . . . 1-4

TASK 2.0 SELECTION OF DANDIDATE MATERIALS ........ 2-1

2.1 Film and Sheet Materials ........... 2-1

2.2 Film From Conductor Coatings . . . . . . . 2-1

2.3 Conductor Coatings and Methods of Application... 2- 2-5

TASK 3.0 EVALUATION OF MECHANICAL CHARACTERISTICS ..... 3-1 Progress ................ 3- . . . . Plans. . . . . . . . . . . . . 3-8

TASK 4.0 EVALUATION OF ELECTRICAL CHARACTERISTICS . . . . 4-1 Progress . . . . . . . . . . . . . . . 4-1 Plans. ................... . . 4-1

TASK 5.0 EVALUATION OF SYSTEM COMPATIBILITY AND AGING . . . 5-1 Progress ................... 5-1 Plans. .................... 5-5

TASK 6.0 EVALUATION OF THERMAL CHARACTERISTICS. ..... 6-

TASK 7.0 TRANSFORMER DESIGN ASSESSMENT. ........ 7-1 


\section{$\underline{\text { Tables }}$}

$\underline{\text { Page }}$

1-1 Range of Dimensions For Sweeping Design Calculations . . . 1-3

2-1 Standard Characteristics of Commercially Available Films $2-2$

2-2 Standard Characteristics of Commercially Available Films . $2-3$

2-3 Films Selected for Investigation .......... 2-4

3-1 Polyester Film Mechanical Properties . . . . . . . . 3-2

3-2 Polyethersulfone Film Mechanical Properties. . . . . 3-3

3-3 Polysulfone Film Mechanical Properties . . . . . . . 3-4

3-4 Aromatic Polyamide Film Mechanical Properties. . . . . . 3-5

3-5 Polyamide Fiber Paper Mechanical Properties. . . . . . 3-6

3-6 Transformer Paper Mechanical Properties (Typical Values) . 3-7

3-7 Conducting Paths and Pinholes in Film Insulation by EFT Test................. 3-. 3

5-1 Dielectric Properties of Mineral 0il for Non-Cellulosic Insulation Program (Water Content: 2 PPM). . . . . 5 5-2

5-2 Dielectric Properties of Silicone Liquid for Non-Cellulosic Insulation Aging Program (Water Content: 6 PPM). . . . .

\section{Figures}

1-1 Flow Chart for Task 1.0. ........... 1-5

5-1 Flat Electrode Test Piece. . . . . . . . 5-4 


\section{SUMMARY}

Work has continued on the development of an analytical model to demonstrate the advantages in both materials and losses to be gained by the utilization of thin insulation in transformers. Measurement of mechanical and electrical properties of film materials is underway. The aging and material compatibility of synthetic films in transformer oil; silicone and RII3 is being studied in a sealed-tube aging program. Several materials are on test and others are being readied for test. 
Task 1

DEVELOPMENT OF PARAMETRIC MODEL FOR SOLID INSULATION IN TRANSFORNERS

\section{Progress}

After reviewing the transformer design programs currently used in General Electric, it was decided early in the quarter that the duration and funding of this project did not permit us to write a new optimization program that would satisfy our needs. For this reason, we decided to make use of existing programs to generate designs of transformers having varying thicknesses of insulation and to explore the effect of changes in other variables in resulting transformer designs. The Distribution Transformer Department's design program, CALOP*, appeared to be the most advantageous for our needs. This is a flexible program that allows the user to select and specify the values of many of the design options. For our purposes one of the most useful features of CALOP is a sweeping mode option. CALOP will sweep through a range of core and coil sizes, giving design results at user specified increments. The range of the sweep is determined by specifying the minimum and maximum dimensions in the input. It is also possible to sweep a range of flux densities: It is possible to select up to five of a possible thirty-two characteristics of interest and specify the maximum and minimum value of each. The choices can include electrical parameters, dimensions or cost related parameters.

By varying the basic insulation level (BIL) value and the operating stress in the program input, design parameters can be obtained for a range of insulation thicknesses in the high voltage (HV) and low voltage (LV) windings. The size of the coils and core as well as the losses in the core and coil vary as the insulation thickness changes. These values are directly related to the cost of materials in the core and coil and to the cost evaluation of the losses. While it is possible to vary the HV insulation and the LV insulation thicknesses independently

ॠCALOP is the name of a General Electric proprietary design program used in the design of distribution transformers. 
over a selected range, for the initial development of model it was decided to express the insulation thickness as a fraction of a reference thickness ( $5 \mathrm{mil}$ for the LV and $10 \mathrm{mil}$ for the HV) and to use as the independent variable the ratio of the insulation thickness to the reference thickness. This ratio has been called $f \Delta x$. When the ratio $f \Delta x$ equals 0.5 for example, the LV insulation is $2.5 \mathrm{mil}$ and the HV insulation is 5 mil.

The variation of insulation thickness $(f \Delta x)$ can be combined with variations of loss evaluation constants (\$/watt). The loss evaluation constants $(K)$ determine the operating cost of the transformer. There are two constants used in the program; one for core or no-load loss and one for load loss. The ratio of core loss evaluation to load loss evaluation is maintained between 1.0 and 1.5 .

For each combination of $f \Delta x$ and $K$ (approximately 5 of each), the sweeping mode option will be used to vary other design parameters listed in Table 1-1 and select the design with the minimum "owner cost". Owner cost is the combination of material cost and operating cost based on losses. This should produce a family of curves showing minimum owner cost as a function of loss evaluation constant and insulation thickness for cellulose insulation.

Since these material costs and the losses are both based on cellulosic insulation, correction for each non-cellulosic insulation can be made based on the cost and thermal properties of the insulation. The material costs can be adjusted by allowing for the difference in cost between paper and the insulation of interest.

$$
\begin{gathered}
\text { Adjusted Cost }=\text { Material Cost in Design + Volume of Paper } x \\
\text { (Unit Cost of Non-Cellulosic - Unit Cost Paper) }
\end{gathered}
$$

The correction for the losses can be made using an existing thermal model which adjusts temperature rise and losses on the basis of the thermal characteristics of the insulation. When these corrections are implemented, the data set will represent the optimum owner cost as a function of insulation thickness and loss evaluation constant for each material. 
Table 1-1

RANGE OF DIMENSIONS FOR SWEEPING DESIGN CALCULATIONS

$\begin{array}{lccc}\text { Feature } & \frac{\text { Min. }}{8.4} & \frac{\text { Max. }}{8.6} & \frac{\text { Increment }}{0.1} \\ \text { Core window height } & 52 & 60 & 2 \\ \text { LV Turns } & 5.6 & 6.7 & 1.1 \\ \begin{array}{l}\text { Core strip width } \\ \text { (wound core) }\end{array} & 0 & 115 & 1 \\ \text { Flux density } & 0 & & \end{array}$


This approach is summarized in Figure 1-1.

\section{Progress}

A program to determine the minimum useful insulation thickness based on mechanical and electrical loading has been written. This should allow the determination of the minimum insulation thickness to give performance comparable to paper.

The input data files for CALOP have been prepared, and the variation of insulation thickness and cost factors has been completed. These results are being tabulated for further analysis.

\section{Future Activity}

The optimization of owner cost through a sweep of dimensions is underway. When this is completed, the correction will be applied for each non-cellulosic material. 


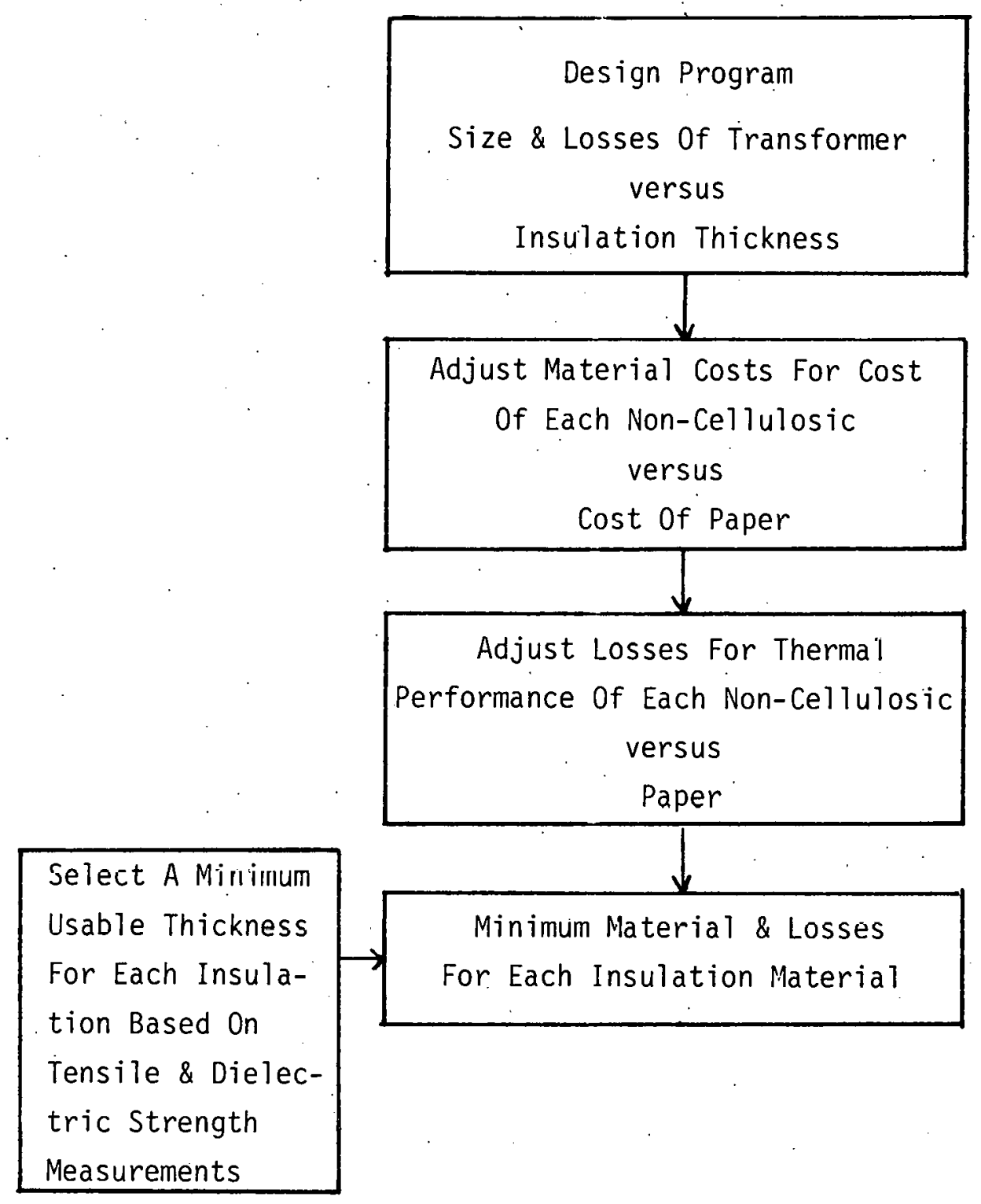

Figure 1-1. Flow Chart for Task 1.0. 
Task 2

SELECTION OF CANDIDATE MATERIALS

\subsection{Film and Sheet Materials}

\section{Progress}

The standard characteristics of commercially available films are summarized in Tables 2-1 and 2-2. These data are taken from the 1979-1.980 Modern Plastics Encyclopedia. Samples of several of these films have been ordered. These are 1 isted in Table 2-3. These materials represent a wide range of polymer types.

\subsection{Film From Conductor Coatings}

Most of the commercially available film materials are based on thermoplastic polymers. Many of these materials are subject to mechanical creep, especially at elevated temperatures. Creepage of this type may limit the range of application of thermoplastic films in transformers. Magnet wire coatings which are either a combination of thermoplastic and thermoset resins or are cross-linked thermoplastics have been designed to have adequate creep resistance. Formvar enamel is a mixture of polyvinylformal (thermoplastic) and a cresol formaldehyde resin (thermoset) is an example of the former type of wire coating. Cross-linked polyethyleneterephthalate is an example of the latter.

Many combinations of thermoplastic and thermo-setting resin systems have been used for solution and powder coating on magnct wire. Some of these may be suitable to make thin thermoset films for transformer application.

\section{Progress}

Films have been made from a combination of polyvinylformal, phenolic and epoxy resin by solution casting from cresol/dichloromethane mixed solvent. The films were formed on a mold reslease treated steel plate at $200^{\circ} \mathrm{C}$ for one hour. The film was removed from the steel plate and heated for 8 to 10 minutes at $250^{\circ} \mathrm{C}$. The film thickness was approximately 50 microns ( $2 \mathrm{mil}$ ). Problems have been encountered in removing the film from the 


\begin{tabular}{|c|c|c|c|c|c|c|c|c|c|c|c|c|c|c|c|c|c|}
\hline \multirow[b]{2}{*}{ Fropertiet } & \multirow[b]{2}{*}{$\mid \begin{array}{l}\text { ASTM } \\
\text { iest } \\
\text { melinod }\end{array}$} & \multicolumn{7}{|c|}{ Fluoroplastics } & \multirow[b]{2}{*}{ Polycnrbonste } & \multirow[b]{2}{*}{$\mid \begin{array}{l}0 \\
\text { Polyester. } \\
\text { ihermoplast c- } \\
\text { polyethylene } \\
\text { perephthalalo } \\
\text { (PET) }\end{array}$} & \multirow[b]{2}{*}{ Polyimide } & \multicolumn{2}{|c|}{ Polypropylene } & \multirow[b]{2}{*}{$\begin{array}{l}\text { Polypara. } \\
\text { banic } \\
\text { ocid } \\
\end{array}$} & \multirow[b]{2}{*}{$\begin{array}{l}\text { Poly- } \\
\text { melthy- } \\
\text { penteno }\end{array}$} & \multirow[b]{2}{*}{ Polysultone } & \multirow[b]{2}{*}{$\begin{array}{l}\text { Polyethor. } \\
\text { P.llone }\end{array}$} \\
\hline & & $\begin{array}{l}\text { Ethyleno- } \\
\text { chlorotri- } \\
\text { fluoro- } \\
\text { ethyllene } \\
\text { copolymer } \\
\text { (E-CTFE) }\end{array}$ & $\begin{array}{l}\text { Eihyleno- } \\
\text { telra- } \\
\text { lluoro- } \\
\text { ethylene } \\
\text { copolyrer } \\
\text { (ETFE) } \\
\end{array}$ & $\begin{array}{l}\text { Fluoringted } \\
\text { ethylene } \\
\text { Fopyleno } \\
\text { Copolymor } \\
\text { (=EP) }\end{array}$ & \begin{tabular}{|l|} 
Polyc loro- \\
trillucro- \\
elhyle ne \\
copofymers \\
(CTFE)
\end{tabular} & 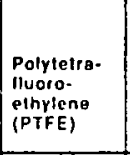 & \begin{tabular}{|l} 
Polyviny: \\
luoride \\
(PVF)
\end{tabular} & $\begin{array}{l}\text { Poly- } \\
\text { vinyl. } \\
\text { idene } \\
\text { lluorice } \\
\text { (PVOF) }\end{array}$ & & & & $\begin{array}{l}\text { Exrtitioion } \\
\text { (cast) }\end{array}$ & $\begin{array}{l}\text { Binxially } \\
\text { oriented } \\
\end{array}$ & & & & \\
\hline 1. Ploduction meinod & - & Extusion & Extrustor & Esilusion & $\begin{array}{l}\text { Cassing. } \\
\text { extiusonon }\end{array}$ & \begin{tabular}{|l} 
Skiving. \\
casting \\
extitusion
\end{tabular} & Exxtusion & Extusion & ן casting. & $\begin{array}{l}\text { Exteusion. } \\
\text { biasiallyonenino }\end{array}$ & - & 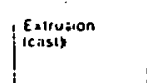 & $\begin{array}{l}\text { Orienlasion } \\
\text { (atsc } \\
\text { cuateded) }\end{array}$ & Casting & $\begin{array}{l}\text { Entrusion. } \\
\text { casting. }\end{array}$ & Extrusion & $\begin{array}{l}\text { Casting. } \\
\text { entursion }\end{array}$ \\
\hline 2 forms ovailable & - & $\begin{array}{l}\text { Rolls, sheets. } \\
\text { lubes }\end{array}$ & $\begin{array}{l}\text { Rolls, shicels. } \\
\text { ludes }\end{array}$ & \begin{tabular}{|l|}
$\begin{array}{l}\text { folls, sheols } \\
\text { lubes }\end{array}$ \\
\end{tabular} & $\begin{array}{l}\begin{array}{l}\text { Aolls, Stcets. } \\
\text { lubing }\end{array} \\
\end{array}$ & \begin{tabular}{|l|} 
Shichif, laces. \\
iwuing \\
\end{tabular} & Poils & Rolis. Siem/s & 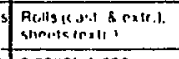 & 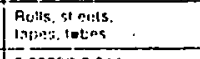 & $\begin{array}{l}\text { Hin's. } \\
\text { infe } \\
\text { infor }\end{array}$ & 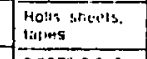 & nollis & Anits & ñons. sneels. & $\begin{array}{l}\begin{array}{l}\text { Rolls. } \\
\text { sheeis }\end{array} \\
\end{array}$ & Polls, stroets \\
\hline 3 Tmenness range. in & - & $0.0005 \cdot 0.090$ & - & .00005 .0036 & 00005.0030 & Upto0030 & $00005 \cdot 0.604$ & $0002-6020$ & $000025,-(1500$ & 0000000014 & 00.075 s.e 005 & 000250010 & 0 (60045-0000\%6 & 0001.0005 & $0003 \cdot 0.030$ & 00001.0030 & 00001.0030 \\
\hline - Maximuer wicth, in. & - & 54 & 48 & 48 & 34 & 48 & 138 & 50 & 45 cast. 54 exir. & 67.120 & 60 & 69 & 72.80 & 52 & 48 & 32 & 32 \\
\hline S. Area lactor (sa. in /10/7nal) & - & 16.600 & 16.300 & 11.900 & 13.000 & 12.800 & 17.200 .20000 & 16.000 & 23.100 & $19.800 \cdot 22.600$ & 19.400 & $30.9=31.300$ & $\begin{array}{l}30.600 \\
\text { (uncoateos) }\end{array}$ & 20.500 & 33.360 & 22.355 & 20.000 \\
\hline 6. Specitic gravaly & Disc5 & $1.66 \cdot 1.66$ & 1.7 & $2 \cdot 15$ & $2.08 \cdot 215$ & $2.1 \cdot 2.2$ & 1.38 .157 & 1.76 & 1.20 & 1.3310 .1410 & .142 & 08950905 & 0902.0907 & 135 & 023 & $124 \cdot 1.25$ & 1.37 \\
\hline 7 Tenste sirengin, p.s.i & 0882 & $8000 \cdot 10.003$ & $7000 \cdot 8000$ & $2500-3000$ & $5000 \cdot 0.000$ & 8500.4500 & $7000 \cdot 18,0.20$ & $5500 \cdot 6500$ & 8000.8800 & $\begin{array}{l}80.900 .35 .000 \\
40.000 \text { ilensilizend } \\
\end{array}$ & 25.000 & $4500 \cdot 10.000$ & 7560.40 .000 & 16.000 & 2700.4000 & $8400 \cdot 10.600$ & $10.000 \cdot 12.000$ \\
\hline EElong̣ation. \% & 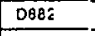 & 150.250 & 300 & 300 & 50.156 & $100 \cdot 350$ & $11,1,200$ & 100.500 & 85.105 & 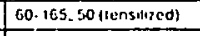 & 70 & 550.000 & 35.475 & 70 & 10 & $60+110$ & 20.150 \\
\hline 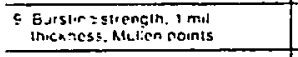 & 0174 & - & 2 & 11 & 23.31 & - & 11970 & & $\begin{array}{l}\text { Noringh } \\
44 \text { mil. } 25.35 \\
\end{array}$ & 50.80 & is & $=$ & - & - & - & $\begin{array}{l}60.51 \\
10002 \cdot \mathrm{mil} \\
\end{array}$ & - \\
\hline O Tearing s:rengin $\mathrm{c} / \mathrm{m} / 0$ & \begin{tabular}{|l|}
$D 19 c^{2}$ \\
\end{tabular} & $900 \cdot 1300$ & 600.900 & 195 & 25.400 & 10.103 & $12 \cdot 100$ & 50 & 20.25 & $12-27$ & 8 & $600.1025 . \mathrm{MD}$ & $3 \cdot 10$ & 8 & & $9-12$ & 7.16 \\
\hline 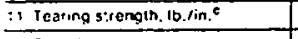 & 01004 & - & - & 600 & 330.900 & - & 0097.400 & & 700.1000 & 1000.3000 & 1120 & $=$ & $1000 \cdot 1500$ & 1000 & 400.650 & 236.243 & 420.950 \\
\hline Z. Foldoring endu:ance & 02136 & $\begin{array}{l}250.000 \\
(1-\mathrm{mill}) \\
\end{array}$ & - & - & Good & - & $5000 \cdot 47.0 .70$ & \begin{tabular}{|l}
$\because 0.040$ \\
(s:mil) \\
\end{tabular} & 250.400 & $\therefore 100.600$ & 10000 & Very $19 n$ & Verf high & 10.000 & 600 & - & - \\
\hline 3 waler absorption $24 \mathrm{hr..} \%$ & 0570 & 4002 & $\because 002$ & $=001$ & Nin. & mit & 105 & 004 & 0.35 & .68 & 29 & .00 .35 & .0005 & 2.8 & 0005 & 0.3 & 2.1 \\
\hline 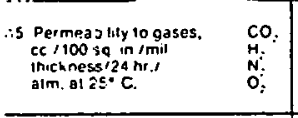 & 01434 & $\frac{110}{10}$ & \begin{tabular}{|l}
230 \\
30 \\
100
\end{tabular} & $\begin{array}{l}1570 \\
2500 \\
320 \\
150 \\
150\end{array}$ & $\begin{array}{l}16.00 \\
220.350 \\
2.5 \\
7.15\end{array}$ & $\begin{array}{l}\bar{z} \\
\bar{z}\end{array}$ & $\mid \begin{array}{ll}111 & 1 \\
58 \\
025 \\
0 & 25\end{array}$ & $\begin{array}{l}55 \\
9 \\
10\end{array}$ & $\begin{array}{l}1075 \\
1600 \\
500 \\
300\end{array}$ & $\begin{array}{l}15.25 \\
100.9 .10 \\
07.1 .0 \\
30.40\end{array}$ & $\begin{array}{l}45 \\
250 \\
65 \\
25\end{array}$ & $\begin{array}{l}500.600 \\
1700 \\
40.46 \\
150-2.40\end{array}$ & $\begin{array}{|ll|}\text { Uncooled } & \text { Cuated } \\
540 & 055.50 \\
50 & 10.20 \\
20 & 05.20 \\
160 & 05.50 \\
160 & 05.50 \\
\end{array}$ & $\bar{z}$ & $\bar{z}$ & $\begin{array}{l}950 \\
\frac{950}{40} \\
!_{230} \\
\end{array}$ & $\begin{array}{l}405 \\
40 \\
90\end{array}$ \\
\hline 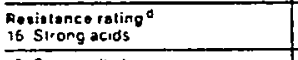 & 0543 & $G$ & 6 & c & $G$ & a & G & G & 6 & 6 & $\mathrm{G}$ & 6 & 6 & 0 & c & 0 & 6 \\
\hline Sirong athalies & 0543 & $G$ & $G$ & $c$ & $G$ & $G$ & 6 & G & : & $\bar{p}$ & $P$ & $-\mathrm{G}$ & $\mathrm{G}$ & $P$ & 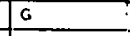 & $\therefore$ & 0 \\
\hline 15. Grease a nd olls & 0722 & 6 & G & $c$ & $a$ & $a$ & is & $\sigma$ & 6 & $a$ & $\mathrm{G}$ & $\bar{c}$ & 6 & $G$ & $F$ & 6 & 6 \\
\hline 19. Orgame solvenis & 0543 & $\bar{G}$ & a & c & $\sigma^{6}$ & 6 & 10 & 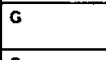 & G10P & o & o & $G$ & $\begin{array}{l}\begin{array}{l}\text { Uncoated G. } \\
\text { Coaleo. attiched }\end{array} \\
\end{array}$ & $G$ & $F$ & $i^{p}$ & 6100 \\
\hline 0 Maler & \begin{tabular}{|l|l|}
$E 96$ \\
\end{tabular} & $G$ & $G$ & $c$ & $G$ & G & ic & $G$ & 6 & 6 & G & $G$ & $G$ & - & $E$ & :a & 6 \\
\hline$\because 1$ Rign celative humidity & 0756 & $G$ & $G$ & $c$ & $G$ & $G$ & ic & 6 & 6 & 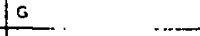 & G & -6 & $G$ & $G$ & $\varepsilon$ & 6 & 6 \\
\hline 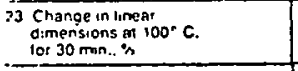 & 01204 & - & - & -1 & $+210-2$ & - & 1 & 02 & ${ }^{\mathrm{Not}}$ & $\therefore 0 s$ & $\begin{array}{l}30 \mathrm{~min} \\
250 \times \\
03: \mathrm{C}^{\circ} \\
03\end{array}$ & - & $\begin{array}{l}\text { Unloer } \\
2 \cdots, \text {. } \\
\text { neal. set }\end{array}$ & $1011200^{\circ} \mathrm{C} .1$ & - & - & 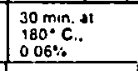 \\
\hline \multicolumn{18}{|l|}{ Eloctrical } \\
\hline 25 Deteectric constant. $1 \mathrm{KHz}$. & 0150 & 2.6 & 2.6 & $: 0.250$ & $23 \cdot 2 .=$ & 20.21 & 85 & 0.4 & 299 & 32 & 35 & 2.2 & 2.2 & 35 & 2.1 & 3.07 & 3.5 \\
\hline 26 Dielectric constant. $1 \mathrm{MHz}^{2}$ & 0130 & 26 & \begin{tabular}{|l|l|}
2.6 \\
\end{tabular} & 0.205 & $2.3 \cdot 2.1$ & 20.2 .1 & 74 & & 293 & 30 & 34 & 2.2 & 2.2 & 34 & $2 \cdot 1$ & 3.03 & 35 \\
\hline 37 Dielectric constant. $16 \mathrm{~Hz}$ & 0150 & - & - & 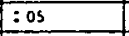 & 23 & 50.71 & $=$ & & 289 & 18 & - & 22 & 22 & $=$ & - & 300 & 3.4 \\
\hline 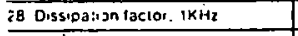 & 0150 & 0002 & 00008 & 00002 & 00220324 & $000<?$ & 16 & 0019 & 00015 & 0000 & 0.0025 & 0.0001 & \begin{tabular}{|l}
0.0002 .00003 \\
\end{tabular} & 00035 & 0003 & 0.0008 & 00035 \\
\hline 39 Oissipa:ion lacior. $1 \mathrm{HHz}$ & 0150 & 0013 & 0.005 & .0004 & 00090517 & 0 one? & 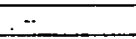 & & 0010 & 0.16 & 0010 & -0.0009 & \begin{tabular}{|l|}
.00002 .000033 \\
\end{tabular} & 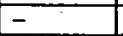 & - & 0.0034 & 0006 \\
\hline 30 Oissivalion faclor, $1 \mathrm{GHz}$ & 0150 & - & - & .0015 & 0004 & 0 outs & $1-$ & & 0012 & 0303.0008 & $=$ & 00008 & .00002 .00003 & - & - & 0.004 & 0.005 \\
\hline 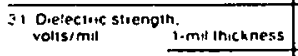 & 0149 & 5000.6000 & 3500 & $=900$ & & $1: 00$ & $i^{3500}$ & & 6300 & $7: 00$ & 2000 & & 8000 & 6300 & & 7500 . & 5800 \\
\hline 5.mil thichness & & & & $: 000$ & & 1000 & 1.2014.mill & & 2000 & 3400 & 3600 & 2700 & & & & & $240014-\mathrm{mill}$ \\
\hline 32 volume esisisyity, ohm-cm. & 0257 & $10^{\circ}$ & $10^{\circ}$ & $0^{\prime \prime}$ & $10^{4}$ & $10^{\circ}$ & $10^{\prime \prime}$ & $10^{\circ}$ & $10^{\circ-}$ & $10^{\prime \prime}$ & $10^{10}$ & $10^{10}$ & $10^{11}$ & $10^{11}$ & $10^{\circ 0}$ & $10^{10}$ & $10^{11} \cdot 10^{4}$ \\
\hline
\end{tabular}




\begin{tabular}{|c|c|c|c|c|c|c|c|c|c|c|c|c|c|c|c|c|}
\hline \multirow{2}{*}{ Table 2-2 } & \multicolumn{7}{|c|}{ Fluorcpi.siics } & \multirow[b]{2}{*}{ Pot, nobonate } & \multirow[b]{2}{*}{ 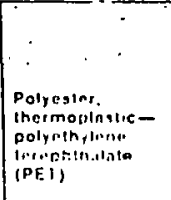 } & \multirow[b]{2}{*}{ Polymido } & \multicolumn{2}{|c|}{ Polypropylono } & \multirow[b]{2}{*}{$\begin{array}{l}\text { polypare. } \\
\text { annic a } \\
\text { ocid }\end{array}$} & \multirow[b]{2}{*}{$\begin{array}{l}\text { poity } \\
\text { latiry. } \\
\text { pentene }\end{array}$} & \multirow[b]{2}{*}{ Polyoultone } & \multirow[b]{2}{*}{ 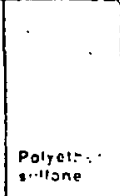 } \\
\hline & 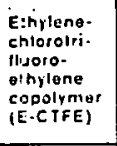 & 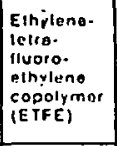 & 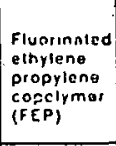 & 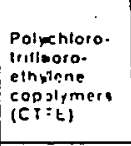 & 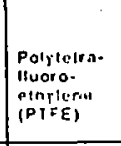 & 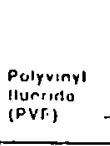 & 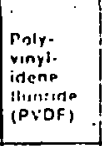 & & & & Ex & 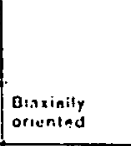 & & & & \\
\hline Crostaline Melt Point ${ }^{\circ} \mathrm{C}$ & 245 & 270 & 200 & 218 & 327 & & 170 & & 260 & & & 165 & & 240 & & \\
\hline $\begin{array}{l}\text { Heat Deflection Temp. } \\
{ }^{\circ} \mathrm{C} / \mathrm{PSI}\end{array}$ & & $71 / 264$ & $70 / 66$ & $125 / 66$ & $60 / 264$ & & $80 / 264$ & $130 / 264$ & $40 / 264$ & $300 / 264$ & & $68 / 264$ & & $40 / 264$ & $275 / 254$ & $200 / 264$ \\
\hline $\begin{array}{c}\text { Temperature Index (UL) } \\
\text { Ccintinuous Use } \\
{ }^{\circ} \mathrm{C}\end{array}$ & .1 .50 & 160 & & & & & & $6 \dot{5}-115$ & 105 & $200-210$ & 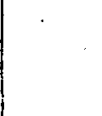 & $80-120$. & $\mid \begin{array}{l}T_{E} \\
290^{\circ} \mathrm{C}\end{array}$ & 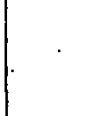 & 140 & 170 \\
\hline Maximum Use Temp., ${ }^{\circ} \mathrm{C}$. & 180 & 180 & $: 250$ & 150 & 250 & 250 & 150 & $i 30$ & 150 & $2 ; 5$ & & 140 & 260 & 205 & 150 & 180 \\
\hline
\end{tabular}


Table 2-3

FILMS SELECTED FOR INVESTIGATION

\begin{tabular}{|c|c|c|}
\hline Material & Thickness (microns) & Status \\
\hline Polyester & 50 & Received \\
\hline Polyethersulfone & $\begin{array}{r}75 \\
100\end{array}$ & $\begin{array}{l}\text { Received } \\
\text { Received }\end{array}$ \\
\hline Polysulfone & 12 & Received \\
\hline Aromatic Polyamide & $\begin{array}{l}10 \\
25 \\
40\end{array}$ & $\begin{array}{l}\text { Received } \\
\text { Received } \\
\text { Received }\end{array}$ \\
\hline Polyamide Fiber Paper & 75 & Received \\
\hline Polyimide & $\begin{array}{r}25 \\
125\end{array}$ & $\begin{array}{l}\text { On Order } \\
\text { On Order }\end{array}$ \\
\hline Polyimide-Thermoplastic & $\begin{array}{l}12 \\
25\end{array}$ & $\begin{array}{l}\text { On Order } \\
\text { On Order }\end{array}$ \\
\hline Polyvinylfluoride & $\begin{array}{r}25 \\
100\end{array}$ & $\begin{array}{l}\text { On Order } \\
\text { On Order }\end{array}$ \\
\hline Polyvinyl idenefluoride & $\begin{array}{l}25 \\
50\end{array}$ & $\begin{array}{l}\text { On Order } \\
\text { On Order }\end{array}$ \\
\hline Polyparabanic Acid & $\begin{array}{r}25 \\
125\end{array}$ & $\begin{array}{l}\text { On Order } \\
\text { On Order }\end{array}$ \\
\hline
\end{tabular}


steel plate and a more effective mold release agent is desirable.

Plans

Sufficient film will be prepared to evaluate its mechanical, electrical and aging characteristics.

One additional polymer system will be investigated.

\subsection{Conductor Coatings and Methods of Application}

The use of thin, uniform, high dielectric strength polymeric film on aluminum would result in a number of benefits in transformer design.

The major problem associated with the coating of thin aluminum sheets is the attainment of adequate dielectric strength at the edges. Conventional techniques like the roller coating and spraying of polymeric solution result in poor edge coverage as a result of surface tension effects which pull the coatings away from the edges during the cure cycle. Electrodeposition of thin polymeric film on the conductor surface offers the advantage of excellent edge coverage prior to cure. Care must be taken, however, in selecting and formulating the system to avoid edge pull-back. Aluminum is especially difficult to coat electrolytically because of its electrochemical passive nature. During deposition, the aluminum is made the anode and immersed in a solution or dispersion of a polymeric material which possesses the negative charge as a result of its inherent structure or as a result of some absorbed ions from solution. On passing $D C$ current through the cell, the following reaction occurs:

$$
2 \mathrm{Al}+3 \mathrm{H}_{2} \mathrm{O} \rightarrow \mathrm{Al}_{2} \mathrm{O}_{3}+6 \mathrm{H}^{+}
$$

with the $\mathrm{H}^{+}$neutralizing the negative charge on the polymer and resulting in the precipitation of the polymer on the aluminum strip. During deposition, the aluminum invariably passivates with part of the current resulting in the following reaction at the aluminum surface:

$$
2 \mathrm{H}_{2} \mathrm{O} \rightarrow 4 \mathrm{H}^{+}+\mathrm{O}_{2}
$$

The $\mathrm{H}^{+}$continues to precipitate polymer on the surface. but the coating develops pinholes and voids as a result of the trapped $0_{2}$ gas. 
It is possible to live with the oxygen evolution by formulating the electro-deposition medium so that the bubbles escape during the cure. of course, extensive flow-out is required during cure. This approach is essentialiy that used in the manufacture of electro-paints. The major problem in this approach is that appreciable edge pull-back occurs with reduced edge coverage relative to the flats.

If excellent edge coverage is required, then a deposit with little or no flow-out is required. If this restriction is put on the system, then one cannot live with $\mathrm{O}_{2}$ evolution and it must be eliminated completely. An approach which seems feasible is the deposition of a more electrochemically active material on the aluminum surface prior to electro-deposition. Zinc deposited from a zincate solution (via immersion) appears to be electrochemically active and might possibly eliminate the evolution of oxygen. The presence of zinc on the aluminum should result in the following electrochemical reaction:

$$
\mathrm{Zn} \rightarrow \mathrm{Zn}^{+\cdot+\cdot+}
$$

with $\mathrm{Zn}^{++}$precipitating the polymer. The effect of $\mathrm{Zn}^{++}$on the aging characteristic of the polymer would have to be investigated as would the optimum condition of zincate treating.

Previous work has centered around the development and application of an electrocoatable amide-imide polymer on aluminum strip. This is a polymer system determined to be adequate for oil/paper insulation systems as well as for dry-type transformers.

While other electrocoatable material systems have heen considered, including electrocoatable acrylics and epoxies, neither hac proven successful for extended life in an $011 /$ paper insulation system. However, the elimination of paper offers the opportunity to consider these materials more seriously. This is true also of electrocoatable ester-imides and recently developed amide-imide polymer suspensions. All of these should be lower in material cost and processing cost than the amide-imides.

\section{Progress}

Electrocoating of conductor with an amide-imide polymer suspension is $2-6$ 
underway. Coating and curing parameters are being explored on a laboratory scale apparatus.

\section{Plans}

Samples of electrocoated conductor will be prepared for mechanical, electrical and aging evaluation.

An attempt will be made to coat aluminum conductor by polymer coagulation techniques. 
Task 3

EVALUATION OF MECHANICAL CHARACTERISTICS

\section{Progress}

The mechanical characteristics have been measured at room temperature for all of the films that have been received. These data are summarized in Tables 3-1 to 3-5. For comparison, the mechanical properties of Kraft paper are summarized in Table 3-6. These measurements were made using standard test procedures, as follows:

$\begin{array}{ll}\text { Thickness } & \text { ASTM D645 } \\ \text { Basis weight } & \text { ASTM D646 } \\ \text { Tear } & \text { ASTM D689 } \\ \text { Burst } & \text { ASTM D774 } \\ \text { Tensile } & \text { ASTM D828 } \\ \text { Elongation } & E=\frac{B-A}{A} \times 100 \\ & \text { where: } A=\text { distance between the jaws at } \\ & \text { the start of the tensile test. } \\ & B=\text { distance between the jaws at } \\ & \text { the moment of break. }\end{array}$

The measurement of the temperature dependence of tensile strength and elongation is underway. These tests are run on a Universal Testing Instrument (Instron), Model TTCM-1, to which an Environmental Chamber, Model A74, is attached. The chamber is set for the desired temperature and allowed to reach equilibrium. The chamber door is opened, a tensile strip of the film under investigation is placed in the jaws of the tester, the door closed, and the chamber and test specimen allowed to reach. equilibrium again (about 10 minutes). A total of five tests per film per temperature are run.

$$
\begin{array}{lll}
\text { Jaws separation } & -5 \text { inches } \\
\text { Jaw speed } & - & 1 \text { inch/min. }
\end{array}
$$

Tensile strength and elongation are recorded on a strip chart. 
POLYESTER FILM MCHANICAL PROPERTIES

Property

Thickness, microns (mils)

Avg.

Max.

Min.

Density, gms/cc

Basis Wt., gms $/ \mathrm{m}^{2}\left(1 \mathrm{bs} / 3000 \mathrm{ft}^{2}\right)$

M.D. Tear, gms. Avg.

Max.

Min.

C.M.D. Tear, gms. Avg.

Max.

Min.

Burst, pascals (psi)

Avg.

Max.

Min.

M.D. Tensile, pascals (psi)

Avg.

Max.

Min.

C.M.D. Tensile, pascals (psi)

Avg.

Max.

Min.
50 Micron

$48.3(1.9)$

$55.9(2.2)$

$43.2(1.7)$

1.43

$68.9(42.3)$

69

71

65

81

83

79 $\begin{array}{ll}140,800 \times 10^{3} & (20,420) \\ 155,700 \times 10^{3} & (22,580) \\ 128,500 \times 10^{3} & (18,630)\end{array}$

$141,200 \times 10^{3}(20,470)$

$147,700 \times 10_{3}^{3}(21,420)$

$122,700 \times 10^{3}(17,790)$

M.D. Elongation, \%

Avg.

Max.

Min.

C.M.D. Elongation, \%

Avg. $\quad 47$

Max. $\quad 52$

Min. 26 
Table 3-2

POLYETHERSULFONE FILM MECHANICAL PROPERTIES

Property

Thickness, microns (mils)

Avg.

Max.

Min.

Density, gms/cc

Basis Wt., gms $/ \mathrm{m}^{2}\left(\right.$ lbs $\left./ 3000 \mathrm{ft}^{2}\right) \quad 104.9(64.4)$

M.D. Tear, gms. Avg.

Max.

Min.

C.M.D. Tear, gms. Avg.

Max.

Min.

Burst, pascals (psi)

Avg.

Max.

Min.

M.D. Tensile, pascals (psi)

Avg.

Max.

Min.

C.M.D. Tensile, pascals (psi)

Avg.

Max.

Min.

M.D. Elongation, \%

Avg.

Max.

Min.

C.M.D. Elongation, \%

Avg.

Max.

Min.
75 Micron

$75.7(3.0)$
$111.8(4.4)$
$55.9(2.2)$

1.4

74

112

57

64

79

53

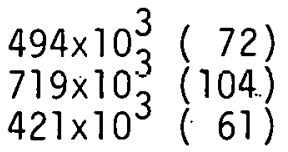

$622 \times 10^{3}\left(\begin{array}{r}90 \\ 707 \times 10^{3}\end{array}(103)\right.$
$524 \times 10^{3}(76)$

114
123
106

114
123
106

109

146

89

100 Micron

$96.5(3.8)$

$116.8(4.6)$

$81.3(3.2)$

1.48

$143.5(88.1)$

$70,410 \times 10^{3}$
$78,930 \times 10^{3}$
$(10,210)$
$63,510 \times 10^{3}\left(\begin{array}{r}17,450) \\ 9,210)\end{array}\right.$

$104,100 \times 10_{3}(15,100)$

$(10,570)$

5.1 
Table 3-3

POLYSULFONE FILM MECHANICAL PROPERTIES

Property

Thickness, microns (mils)

Avg.

Max.

Min.

Density, gms/cc

Basis $W t ., g m s / \mathrm{m}^{2}\left(1 \mathrm{bs} / 3000 \mathrm{ft}^{2}\right)$

M.D. Tear, gms. Avg.

Max.

$M+n$.

C.M.D. Tear, gms. Avg.

Max.

Min.

Burst, pascals (psi)

Avg.

Max.

Min.

M.D. Tensile, pascals (psi)

$\wedge$ vg.

Max.

Min.

C.M.D. Tensile, pascals (psi)

Avg.

Max.

Min.

M.D. Elongation, \%

Avg.

Max.

Min.

C.M.D. Elongation, \%

Avg.

Max.

Min.
12 Micron

$11.7(0.46)$

$12.7(0.50)$

$11.1(0.44)$

$$
2.2
$$

$16.8(10.3)$

9.7

14

8

9.8

11

9

$\begin{array}{ll}159 \times 10^{3} & (23) \\ 165 \times 10^{3} & (24) \\ 152 \times 10^{3} & (22)\end{array}$

$67,450 \times 10_{3}^{3}(9780)$

$83,940 \times 10^{3}(12170)$

$56,960 \times 10^{3}(8260)$

$55,460 \times 10^{3}(8040)$

$59,900 \times 10.3(8700)$

$50,960 \times 10^{3}(7390)$
6.7

9.6

3.7

4.6

6.0

3.4 
Table 3-4

AROMATIC POLYAMIDE FILM MECHANICAL PROPERTIES

Property

Thickness, microns (mils)

$$
\text { Avg. }
$$

Min.

Density, gms/cc

Basis Wt., gms $/ \mathrm{m}^{2}\left(1 \mathrm{bs} / 3000 \mathrm{ft}^{2}\right)$

M.0. Tear, gms.

Avg.
Max.

Min.

C.M.D. Tear, gms. Avg.

Max.

Min.

$\omega$ Burst, pascals (psi)

o

Avg.

\section{Micron

$$
\begin{array}{r}
10.80(0.43) \\
9.50(0.38)
\end{array}
$$$$
9.90(0.39)
$$

Max.

Min.

M.D. Tensile, pascals (psi)

$$
\text { Avg. }
$$

Max.

Min.

C.M.D. Tensile, pascals (psi)

$\begin{array}{ll}\text { Avg. } & 122,000 \times 10^{3}(17,700) \\ \text { Max. } & 127,300 \times 10^{3}(18,500) \\ \text { Min. } & 118,400 \times 10^{3}(17,200)\end{array}$

$$
1.40
$$

$13.8(8.5)$

$$
\begin{array}{r}
9 \\
10 \\
9
\end{array}
$$

$$
9
$$$$
9
$$$$
9
$$

25 Micron

$$
\begin{aligned}
& 22.70(0.90) \\
& 24.50(0.96) \\
& 21.60(0.85)
\end{aligned}
$$

$$
1.30
$$

$28.2(17.3)$

\section{6}

17
15

\section{7}

18

16

$$
\begin{array}{ll}
340 \times 10^{3} & (50) \\
366 \times 10^{3} & (53) \\
329 \times 10^{3} & (48)
\end{array}
$$

$122,000 \times 10_{3}^{3}(17,700)$

$134,400 \times 10^{3}(19,500)$

$114,900 \times 10^{3}(16,700)$

$121,700 \times 10_{3}^{3}(17,600)$

$131,000 \times 10^{3}(19,000)$

$109,400 \times 10^{3}(15,900)$

$122,500 \times 10^{3}$
$126,300 \times 10^{3}(17,800)$
$117,900 \times 10^{3}(17,300)$
40 Micron

$40.2(1.58)$

$41.0(1.61)$

$39.4(1.55)$

1.35

$54.6(33.5)$

25

27

24

25

26

25

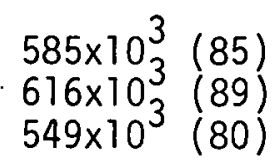

$136,500 \times 10_{3}^{3}(19,800)$ $142,200 \times 10_{3}^{3}(20,600)$ $131,700 \times 10^{3}(19,100)$

$147,800 \times 10_{3}^{3}(21,400)$ $157,400 \times 10^{3}(22,800)$ $143,000 \times 10^{3}(20,700)$

M.D. Elongation, \%

Avg.

Max.

Min.

C.M.D. Elongation, \%

$$
\begin{array}{r}
9.6 \\
11.6
\end{array}
$$$$
5.0
$$

Avg.

Max.

Min.
9.6
14.0
7.6

$$
\begin{array}{r}
9.3 \\
10.0
\end{array}
$$

8.4

10.5

12.9

8.1
14.1

18.1

11.4

12.3 
Table 3-5

POLYAMIDE FIBER PAPER MECHNICAL PROPERTIES

Property

Thickness, microns (mils)

Avg.

Max.

Min.

Density, gms/cc

Basis $w t ., g m s / \mathrm{m}^{2}\left(1 \mathrm{bs} / 3000 \mathrm{ft}^{2}\right)$

M.D. Tear, gms. Avg.

Max.

Min.

C.M.D. Tear, gms. Avg.

Max.

Min.

Burst, pascals (psi)

Avg.

Max.

Min.

M.D. Tensile, pascals (psi)

Avg.

Max.

Min.

C.M.D. Tensile, pascals (psi)

Avg.

Max.

Min.

M.D. Elonqation, \%

Avg.

Max.

Min.

C.M.D. Elongation, \%

Avg.

Max.

Min.

\section{Micron}

$71.1(2.8)$

$81.3(3.2)$

$66.0(2.6)$

0.72

$51.0(31.3)$

98
103
94

146

169

114

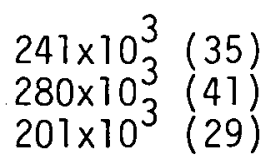

$59,810 \times 10_{3}^{3}(8680)$

$63,780 \times 10_{3}^{3}(9250)$

$54,670 \times 10^{3}(7930)$

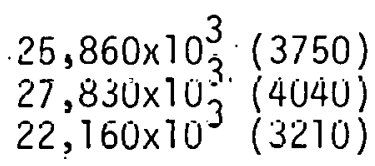

6.5

8.4

4.4

4.6

6.0

2.9 


\section{Property}

Thickness, microns (mils)

Avg.

Max.

Min.
125 Micron

$129.5(5.1)$

Density, gms/cc

1.02

Basis Wt., gms $/ \mathrm{m}^{2} \cdot\left(1 \mathrm{bs} / 3000 \mathrm{ft}^{2}\right)$

$132(81.3)$

M.D. Tear, gms. Avg. Max.

133

Min.

C:M.D. Tear, gms. Avg.

Max.

Min.

Burst, pascals (psi)

Avg.

Max.

$591 \times 10^{3}(86)$

Min.

M.D. Tensile, pascals (psi)

Avg.

Max.

$140,600 \times 10^{3}(20,392)$

Min.

C.M.D. Tensile, pascals (psi)

Avg.

Màx.

Min.

M.D. Elongation, \%

Avg.

2.0

Max.

Min.

C.M.D. Elongation, \%

Avg.

5.7

Max.

Min. 
The number of pinholes or conducting particles in the films has been measured using an Electrical Formation Test (EFT). The Electrical

Formation Tester consists of a brass plate electrode, $14^{\prime \prime} \times 26^{\prime \prime}$ with a smoothly machined surface; a hand roller (with an insulated handle), one-inch wide and two inches in diameter; a six-lane slotted insulation board to provide one square foot of test area; and, the necessary electrical components to vary the voltage from 0 to 5,000 volts $D C$.

It was designed originally to measure electrically the formation (pinholes and conducting particles) of capacitor tissue. It also has been used to test polymeric films.

The brass plate is covered with the material to be tested and the slotted board positioned on top. The voltage is raised to. 100 volts and the roller is passed over the test specimen. After each pass, the voltage is increased in 100-volt increments until the first break occurs. The voltage at which the first break occurred is recorded. The results of this test are summarized in Table 3-7.

Plans

The measurement of the physical and mechanical properties will be continued as additional samples become available.

When all of the data are complete, they will be summarized in a matrix table for easier comparison of all the materials. 
Table 3-7

CONDUCTING PATHS AND PINHOLES IN FILM INSULATION BY EFT TEST

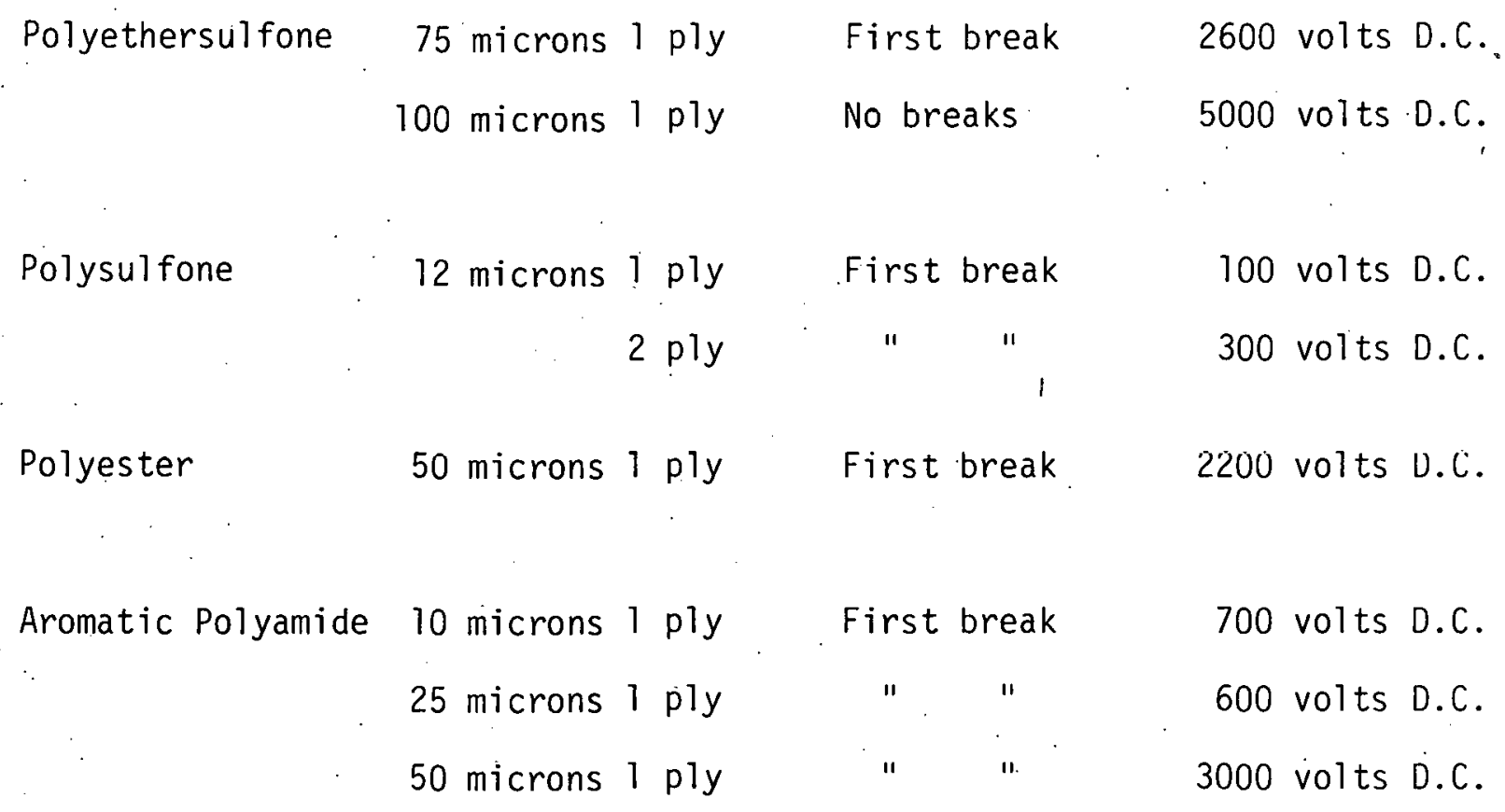


Task 4

EVALUATION OF ELECTRICAL CHARACTERISTICS

\section{Progress}

Preliminary $60-\mathrm{Hz}$ breakdown tests were made in air on several of the. candidate films in order to establish safe upper limits of voltage stress for the dielectric measurements. As a result of these tests, which were made on polyamide fiber paper and polyethersulfone film, it has been decided to make $60-\mathrm{Hz}$ dielectric measurements (on these materials, at least) at voltage stresses of $100,200,500$ and 1000 volts per mil. Measurements of $D C$ volume resistivity will be made at voltage stresses of 100,500 and 1000 volts per mil. Both the $60-\mathrm{Hz}$ and $\mathrm{DC}$ measurements will be made at room temperature, 60,100 and $125^{\circ} \mathrm{C}$, in both air and transformer oil. These measurements, which will be made using a rigid electrode system, will be supplemented by $60-\mathrm{Hz}$ measurements using the fluid-displacement method described in ASTM.DI50. The technique to be emplioyed for the $60-\mathrm{Hz}$ measurements on the remaining film insulations will be determined on the basis of the results obtained on the initial two materials evaluated.

Plans

The dielectric measurements will be completed on poiyethersulfone, polysulfone; and polyester film and a polyamide fiber paper. Additional materials will be tested as they are received.

Breakdown values (60-Hz and impulse) will be determined for all the materials. 
Compatibility and aging tests are designed to determine the interaction of materials and to ascertain the extent to which one material will be likely to impair the performance of others. These tests provide a measure of the insulation system's ability to function over extended periods of time.

Work with cellulosic insulation systems has led to the development of sealed tube test methods which simulate the performance of materials in life test transformers. While the extension of these tests to synthetic solids has not been investigated, the sealed tube evaluation should provide a means of prescreening these materials. The results of these tests can be evaluated by comparison to the extensive data-base of paper/mineral oil systerns.

\section{Progress}

Standard aging tubes are underway at $175^{\circ} \mathrm{C}$ and $160^{\circ} \mathrm{C}$. These tubes contain 8 grams of film material (pre-cut tensile strips), 6 wire pairs with Formvar enamel on copper, one piece of $1.27 \mathrm{~cm} \times 17.8 \mathrm{~cm}$ core steel, and $72 \mathrm{ml}$ of degassed transformer liquid. Tubes containing transformer mineral $0 i 1$ and silicone are under test. The electrical properties of the mineral $0 i 1$ are summarized in Table 5-1 and those of the silicone are summarized in Table 5-2. There are four tubes for each material combination at each temperature. Films under test thus far include polyester, polyethersulfone, and polyamide fiber paper. Tubes being prepared for test include polysulfone and aromatic polyamide films.

In order to measure the change in dielectric characteristics as a function of time, flat electrode test pieces (Figure $5-1$ ) have been designed and constructed. An attempt to use a miniature coil structure was not successful due to the inability to find a suitable non-cellulose winding form for the test coil. Flat electrode pieces have been used to test 
Table 5-1

DIELECTRIC PROPERTIES OF MINERAL OIL FOR NON-CELLULOSIC INSULATION PROGRAM (WATER CONTENT: 2 PPM)

\begin{tabular}{|c|c|c|c|c|c|c|c|c|c|}
\hline \multirow{2}{*}{$\begin{array}{l}\text { Temp. } \\
\text { ( c) }\end{array}$} & \multicolumn{5}{|c|}{$\frac{60-\mathrm{Hz} \text { Dissipation Factor }-\%}{\text { Volts/mil }}$} & \multirow{2}{*}{$\begin{array}{l}60-\mathrm{Hz} . \\
\text { Diel. } \\
\text { Const. }\end{array}$} & \multicolumn{3}{|c|}{$\begin{array}{c}\text { D-C Volume Resistivit } \\
\left(\times 10^{12} \text { ohm- } \mathrm{cm}\right) \\
\text { Volts/mil }\end{array}$} \\
\hline & 5 & 10 & 20 & 40 & 50 & & 10 & 40 & 80 \\
\hline 24 & .009 & .010 & .011 & .012 & .012 & 2.17 & 4330 & 7980 & 8740 \\
\hline 60 & .042 & .042 & .043 & .046 & .048 & 2.10 & 502 & 1020 & 1320 \\
\hline 99 & .126 & .130 & .139 & .153 & .162 & 2.03 & 59.8 & 103 & 123 \\
\hline 123 & .265 & .272 & .284 & .324 & .343 & 1.99 & 17.4 & 28.7 & 32.4 \\
\hline 60 & .030 & .034 & .035 & .039 & .040 & 2.10 & 165 & 219 & 223 \\
\hline 23 & .006 & .007 & .008 & .009 & .010 & 2.17 & 1140 & 1620 & 1860 \\
\hline
\end{tabular}


Table 5-2

DIELECTRIC PROPERTIES OF SILICONE LIQUID FOR NON-CELLULOSIC INSULATION AGING PROGRAM (WATER CONTENT: 6 PPM)

\begin{tabular}{|c|c|c|c|c|c|}
\hline \multirow[b]{2}{*}{ Property Measured } & \multicolumn{5}{|c|}{$\begin{array}{l}\text { Property at Electrical Stresses of } 5 \text {, } \\
\text { Temp. } 10,20 \text { and } 40 \text { volts/mil }\end{array}$} \\
\hline & $\left({ }^{0} \mathrm{C}\right)$ & 5 & 10 & 20 & 40 \\
\hline $\begin{array}{l}60-\mathrm{Hz} \text { Dissipation } \\
\text { Factor }-\%\end{array}$ & $\begin{array}{r}23 \\
61 \\
100 \\
124 \\
100 \\
61 \\
23\end{array}$ & $\begin{array}{r}<.001 \\
<.001 \\
.005 \\
.016 \\
.006 \\
<.001 \\
<.001\end{array}$ & $\begin{array}{r}\leq .001 \\
.003 \\
.010 \\
.019 \\
.009 \\
<.001 \\
<.001\end{array}$ & $\begin{array}{r}.003 \\
.007 \\
.017 \\
.025 \\
.011 \\
<.001 \\
\sim .002\end{array}$ & $\begin{array}{r}.012 \\
.015 \\
.021 \\
.026 \\
.017 \\
.001 \\
.009\end{array}$ \\
\hline $\begin{array}{l}\text { 60-Hz Dielectric } \\
\text { Constant }\end{array}$ & $\begin{array}{r}23 \\
61 \\
100 \\
124 \\
100 \\
61 \\
23\end{array}$ & $\begin{array}{l}2.73 \\
2.59 \\
2.47 \\
.239 \\
2.45 \\
2.58 \\
2.72\end{array}$ & $\begin{array}{l}2.73 \\
2.59 \\
2.47 \\
2.39 \\
2.45 \\
2.58 \\
2.72\end{array}$ & $\begin{array}{l}2.73 \\
2.59 \\
2.47 \\
2.39 \\
2.45 \\
2.58 \\
2.72\end{array}$ & $\begin{array}{l}2.73 \\
2.59 \\
2.47 \\
2.39 \\
2.45 \\
2.58 \\
2.72\end{array}$ \\
\hline $\begin{array}{l}\text { D-C Volume Resistivity* } \\
\left(\times 10^{12} \mathrm{ohm}-\mathrm{cm}\right)\end{array}$ & $\begin{array}{r}23 \\
61 \\
100 \\
124 \\
100 \\
61 \\
23\end{array}$ & $\begin{array}{l}- \\
- \\
- \\
- \\
- \\
-\end{array}$ & $\begin{array}{r}1400 \\
600 \\
230 \\
150 \\
250 \\
560 \\
1300\end{array}$ & $\begin{array}{r}370 \\
160 \\
110 \\
96 \\
140 \\
210 \\
360\end{array}$ & $\begin{array}{r}180 \\
95 \\
82 \\
87 \\
100 \\
100 \\
100\end{array}$ \\
\hline : & . & & & & \\
\hline
\end{tabular}




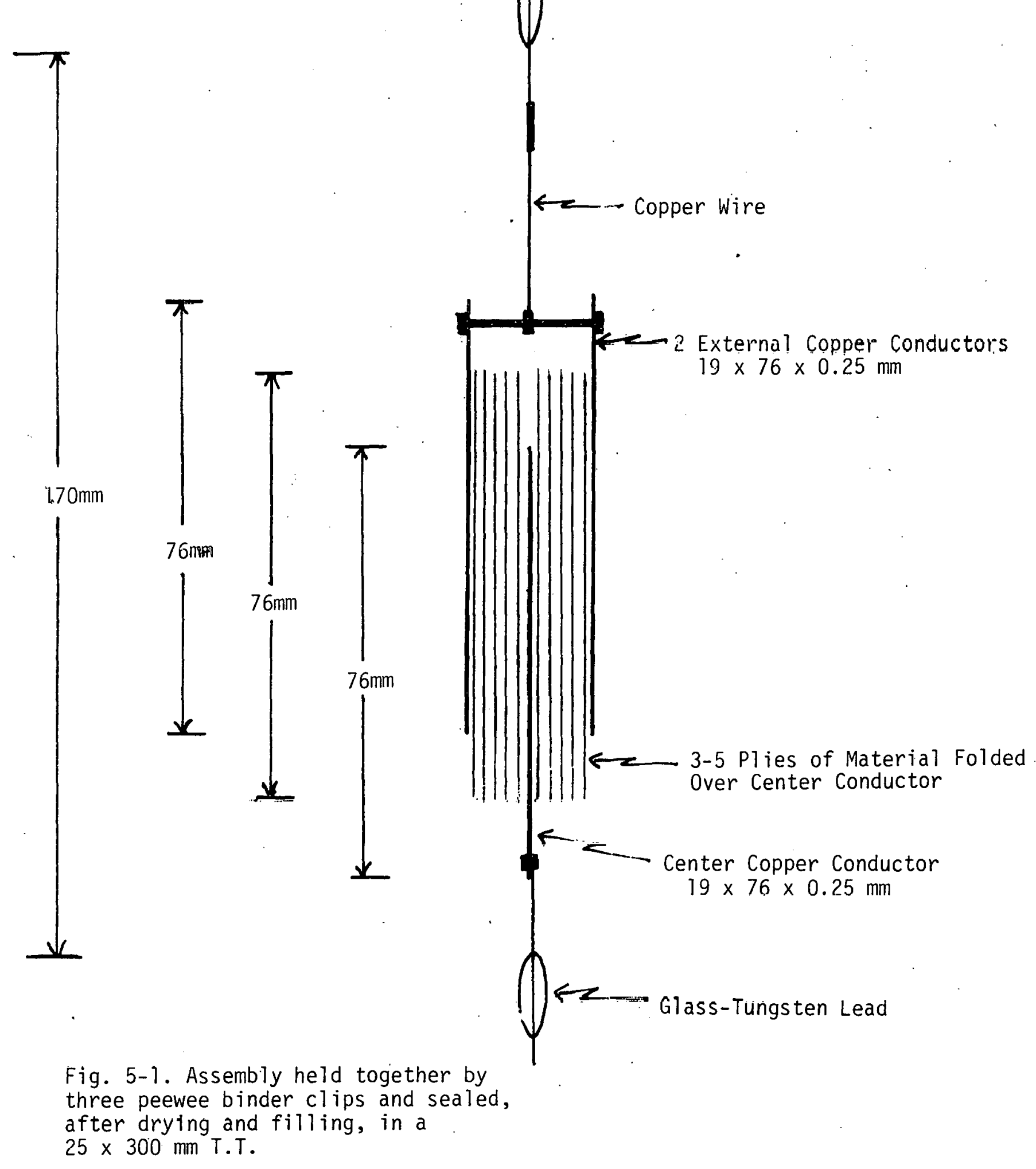


cellulose aging, especially when it is desirable to increase the quantity of insulation between the electrodes.

Test pieces assembléd and ready for test include polyester film, polyethersulfone film, and polysulfone film. Each of these in transformer oil, silicone liquid and R113 (trichlorotrifluoroethane) for a total of nine insulation systems. All oil and silicone samples will be aged at $175^{\circ} \mathrm{C}$. The R113 samples will be aged at $145^{\circ} \mathrm{C}$.

An attempt to seal test samples in $\mathrm{SF}_{6}$ failed because of the breakdown of $\mathrm{SF}_{6}$ at molten glass temperature resulting in the etching of the glass by $H F$.

Plans

The tubes on test will be continued for the planned time intervals.

Tubes in preparation will be placed on test when they are ready. Additional test pieces will be assembled and tested, as the materials become available. 


\section{Task 6}

EVALUATION OF THERMAL CHARACTERISTICS

No work planned during second Quarter. 
Task 7

TRANSFORMER DESIGN ASSESSMENT

No work planned during second Quarter. 